\title{
Адсорбционные характеристики материалов ограждающих конструкций
}

\author{
И.Я.Киселёв, НИИСФ РААСН, Москва
}

Знание численных значений адсорбционных характеристик материалов ограждающих конструкций зданий необходимо для проведения расчётов теплотехнических показателей этих конструкций. Адсорбционными характеристиками материала являются: константа с уравнения Брунауэра-Эммета-Тейлора (БЭТ) сорбции паров воды, равновесная сорбционная влажность $w_{\mathrm{m}}$ материала, соответствующая заполнению первого монослоя адсорбированных молекул воды (ёмкость монослоя), относительной влажности воздуха $\varphi_{\mathrm{m}}$, соответствующей заполнению первого монослоя, и удельная поверхности $A$ материала. Существует два метода обработки изотерм сорбции паров воды исследуемым материалом с целью определения этих характеристик. Первый метод основан на использовании уравнения БЭТ. Второй метод, названный $N$-метод, представляет собой модификацию первого.

Уравнение БЭТ применимо для анализа изотермы сорбции только в диапазоне относительных давлений адсорбата от 0,05 до 0,35. N-метод применим для анализа изотермы сорбции в диапазоне относительных влажности воздуха от 0,05 до 0,5. Таким образом, основное преимущество N-метода по сравнению с методом, основанным на уравнении БЭТ, заключается в том, что N-метод позволяет получить более точную информацию об адсорбционных характеристиках исследуемого материала, так как эту информацию получают путём обработки большего числа экспериментально полученных значений равновесной сорбционной влажности этого материала.

С целью сопоставления двух вышеупомянутых методов по этим методам были обработаны полученные на вакуумной сорбционной установке при температуре $+20{ }^{\circ} \mathrm{C}$ изотермы сорбции восьми строительных материалов: цементного камня, тяжёлого бетона, керамзитобетона, азеритобетона, красного кирпича, цементно-песчаного раствора и пенобетона. По результатам обработки были определены адсорбционные характеристики и получены уравнения начальных участков изотерм сорбции исследованных материалов при температуре $+20^{\circ} \mathrm{C}$.

Ключевые слова: строительные материалы, адсорбционные характеристики, методы определения, сопоставление.

\section{The Adsorption Characteristics of the Enclosing Construction Materials}

\section{I.Ya.Kiselev, NIISF RAASN, Moscow}

The knowledge of the numerical values of the adsorption characteristics of the materials of the building envelope is necessary for the calculation of the thermal performance of these structures. The adsorption characteristics of the material are: constant c of the Brunauer-Emmett-Taylor equation (BET) of the water vapor sorption, the equilibrium sorption moisture $w_{\mathrm{m}}$ of the material, corresponding to the filling of the first monolayer of adsorbed water molecules (monolayer capacity), relative air humidity $\varphi_{\mathrm{m}}$, corresponding to the filling of the first monolayer, and specific surface $A$ of the material. There are two methods for processing isotherms of sorption of water vapor by the studied material in order to determine these characteristics. The first method is based on the use of the BET equation. The second method, called the $\mathrm{N}$-method, is a modification of the first.

The BET equation is applicable for analysis of the sorption isotherm only in the range of relative adsorbate pressures from 0.05 to 0.35 . The $\mathrm{N}$-method is applicable for the analysis of sorption isotherms in the range of relative air humidity from 0.05 to 0.5 . Thus, the main advantage of the $\mathrm{N}$-method compared to the method based on the BET equation is that the $\mathrm{N}$-method allows one to obtain more accurate information on the adsorption characteristics of the material under study, since this information is obtained by processing alarger number of experimentally obtained equilibrium values sorption humidity of this material.

In order to compare the two above-mentioned methods, these methods were used to process the sorption isotherms of eight building materials obtained in a vacuum sorption installation at a temperature of $+20^{\circ} \mathrm{C}$ : cement stone, heavy concrete, expanded clay concrete, azerit concrete, red brick, cement-sand mortar and foam concrete. Based on the processing results, the adsorption characteristics were determined and the equations of the initial sections of the sorption isotherms of the studied materials were obtained at a temperature of $+20^{\circ} \mathrm{C}$.

Keywords: Construction materials, adsorption characteristics, methods of determination, comparison.

\section{Методы обработки изотерм сорбции}

Знание численных значений адсорбционныххарактеристик материалов ограждающих конструкций зданий необходимо для проведения расчётов сопротивления теплопередаче этих конструкций [1-6]. Существует два метода обработки изотерм сорбции паров воды материалами с целью определения константы $c$ исследуемого материала, равновесной сорбционной влажности $w_{\mathrm{m}}$ материала, соответствующей заполнению 
первого монослоя адсорбированных молекул воды (ёмкость монослоя), относительной влажности воздуха $\varphi_{\mathrm{m}}$, соответствующей заполнению первого монослоя, и удельной поверхности $A$ материала. Первый метод основан на использовании уравнения Брунауэра-Эммета-Тейлора (БЭТ) [7] при обработке изотермы сорбции исследуемого материала. Второй метод, разработанный В.Г. Гагариным [8] и названный им N-метод, представляет собой модификацию первого.

До начала описания метода, основанного на использовании уравнения БЭТ, определим связь между значением относительной влажности воздуха $\varphi_{\mathrm{m}}$, соответствующей заполнению первого монослоя, и константой с уравнения БЭТ. Запишем уравнение сорбции водяного пара материалом в виде уравнения БЭТ:

$$
w_{p}(\varphi)=\frac{w_{m} \varphi}{(1-\varphi)\left(\frac{1}{c}+\frac{c-1}{c} \varphi\right)},
$$

где $w_{\mathrm{p}}$ - равновесная сорбционная влажность материала, кг/ кг; $w_{\mathrm{m}}$ - ёмкость монослоя, кг/кг; $\varphi$ - относительная влажность воздуха, Па/Па; $c$ - константа уравнения БЭТ.

Очевидно, что если $\varphi=\varphi_{\mathrm{m}}$, то $w_{\mathrm{p}}=w_{\mathrm{m}}$, тогда уравнение (1) принимает вид:

$$
\frac{\varphi_{m}}{\left(1-\varphi_{m}\right)\left(\frac{1}{c}+\frac{c-1}{c} \varphi_{m}\right)}=1 .
$$

Уравнение (2) приводится к квадратному уравнению, решение которого при $c \geq 1$ имеет вид:

$$
\varphi_{m}=\frac{1}{1+\sqrt{c}}
$$

Следует подчеркнуть, что $c \geq 1$ для всех строительных материалов, исследованных в данной работе, а также в работах В.Г. Гагарина [8] Т.А. Литвиновой [9] и Дж.В Уолен (J.W.Whalen) [10].

При обработке изотермы исследуемого материала по первому методу, основанному на использовании уравнения БЭТ, запишем это уравнение в следующем виде:

$$
\frac{\varphi}{w_{p}(1-\varphi)}=\frac{c-1}{w_{m} c} \varphi+\frac{1}{w_{m} c} .
$$

При этой форме записи $y=\frac{\varphi}{w_{p}(1-\varphi)}$ является линейной функцией аргумента $x=\varphi$. Далее для исследуемого материала по $n$ измеренным значениям его равновесной сорбционной влажности $w_{\text {рi }}$, которые соответствуют значениям относительной влажности воздуха $\varphi_{\mathrm{i}}$, составляем таблицу значений $y_{i}=\frac{\varphi_{i}}{w_{p i}\left(1-\varphi_{i}\right)}$, соответствующих $x_{\mathrm{i}}=\varphi_{\mathrm{i}}$. На основании данных, представленных в этой таблице, методом регрессивного анализа находим коэффициенты линейной регрессии:

$$
\begin{array}{ll}
\text { угловой } & a_{5}=\frac{c-1}{w_{m} c} \\
\text { и постоянной } & b_{5}=\frac{1}{w_{m} c}
\end{array}
$$

Из уравнений (5) и (6) следует:

$$
\begin{gathered}
w_{m}=\frac{1}{a_{5}+b_{5}}, \\
c=\frac{a_{5}+b_{5}}{b_{5}}=\frac{a_{5}}{b_{5}}+1 .
\end{gathered}
$$

Вычисляем по формулам (3), (7) и (8) значения ёмкости монослоя $w_{\mathrm{m}^{\prime}}$ константы с и относительной влажности воздуха $\varphi_{\mathrm{m}}$. Подставив вычисленные значения $w_{\mathrm{m}}$ и $c$ в формулу (1), получим уравнение изотермы сорбции исследуемого материала при той температуре, при которой проведён эксперимент. Далее вычисляем удельную поверхность $A$ исследуемого материала по формуле :

$$
A=w_{m} \frac{N_{A} A_{m}}{M},
$$

где $A$ - удельная поверхность, м²/кг; $A_{\mathrm{m}}$ - площадь, занимаемая одной адсорбированной молекулой воды на поверхности пор материала, м²; и $M$ - молярная масса воды, кг/кмоль. $A_{\mathrm{m}}$ - параметр, связывающий ёмкость монослоя с удельной поверхностью материала.

Он не равен площади поперечного сечения молекулы воды, хотя непосредственно связан с этой величиной. Поэтому для определения этого параметра следует применять термин «эффективная площадь поперечного сечения молекулы воды», а не термин «площадь поперечного сечения молекулы воды».

При наиболее плотной упаковке молекул воды, образующих первый адсорбированный монослой на поверхности пор, $A_{\mathrm{m}}=10,6 \cdot 10^{-20} \mathrm{~m}^{2}$ [7]. Согласно обзору работ, в которых измерялось значение $A_{\mathrm{m}}$ для молекул воды, $A_{\mathrm{m}}=10,6 \cdot 10^{-20} \ldots$

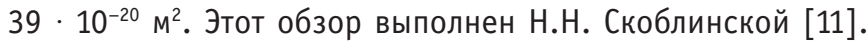
В данной работе принято, что при температуре $+20{ }^{\circ} \mathrm{C}$ для молекул воды $A_{\mathrm{m}}=11,4 \cdot 10^{-20} \mathrm{~m}^{2}$. Это значение заимствовано из работы С. Брунауэра [7], в которой данное значение $A_{\mathrm{m}}$ получено по результатам исследования адсорбции паров воды на тобирморите - материале, который по своему химическому составу близок к исследованным строительным материалам.

При обработке изотермы исследуемого материала по $N$-методу запишем уравнение изотермы в следующем виде:

где

$$
\begin{gathered}
\frac{w_{p}}{N(\varphi, T)}=-\frac{1}{c-1} \cdot \frac{w_{p}}{\varphi \cdot N(\varphi, T)}+\frac{w_{m} c}{c-1}, \\
N(\varphi, T)=\left(\frac{a^{*}}{-\ln \varphi}\right)^{0,322}, \\
a^{*}=\left\{\begin{array}{cc}
\frac{7,84 \cdot 10^{6}}{R T} \sqrt{\frac{\varphi}{0,1}} & \text { при } 0<\varphi<0,1 \\
\frac{7,84 \cdot 10^{6}}{R T} & \text { при } 0,1 \leq \varphi \leq 0,85 \\
\frac{7,84 \cdot 10^{6}}{R T} \sqrt{\frac{1-\varphi}{0,85}} & \text { при } 0,85<\varphi<1
\end{array}\right.
\end{gathered}
$$

При этой форме записи $y=\frac{w_{p}}{N}$ является линейной функцией аргумента $x=\frac{w_{p}}{N \cdot \varphi}$. Далее для исследуемого материала по $n$ измеренным значениям его равновесной сорбционной влажности $w_{\text {рi }}$, которые соответствуют значениям относительной влажности воздуха $\varphi_{\mathrm{i}}$, составляем таблицу значений $y_{i}=\frac{w_{p i}}{N}$, 
соответствующих $x_{i}=\frac{w_{p i}}{N \cdot \varphi_{i}}$. На основании данных, представленных в этой таблице, методом регрессивного анализа находим коэффициенты линейной регрессии:

$$
\begin{aligned}
& \text { угловой } a_{N}=-\frac{1}{c-1} \\
& \text { и постоянный } b_{N}=\frac{w_{m} c}{c-1} . \\
& \text { Из уравнений (13) и (14) следует: } \\
& w_{m}=\frac{b_{N}}{1-a_{N}}, \\
& c=\frac{a_{N}-1}{a_{N}} .
\end{aligned}
$$

Вычисляем по формулам (15) и (16) значения ёмкости монослоя $w_{\mathrm{m}}$ и константы $c$. Подставив вычисленные значения $w_{\mathrm{m}}$ и $c$, а также $N(\varphi, T)$ согласно (11) в формулу (10), получим уравнение изотермы сорбции исследуемого материала при той температуре, при которой проведён эксперимент. Для удобства последующего анализа целесообразно записать уравнение изотермы сорбции в виде:

$$
w_{p}(\varphi)=\frac{w_{m} \cdot c \cdot \varphi}{1+(c-1) \varphi} N(\varphi) .
$$

Далее вычисляем удельную поверхность исследуемого материала по формуле (9).

Остановимся подробнее на определении значения относительной влажности воздуха $\varphi_{\mathrm{m}}$ при обработке изотермы по $N$-методу. Подставив в уравнение (17) $w_{\mathrm{p}}=w_{\mathrm{m}}$ и $\varphi=\varphi_{\mathrm{m}}$ получим следующее уравнение относительно $\varphi_{\mathrm{m}}$ :

$$
\varphi_{m}=\frac{1}{c\left(\frac{a^{*}}{-\ln \varphi_{m}}\right)^{0,392}-(c-1)} .
$$

Не представляется возможным найти аналитическое решение уравнения (18) относительно $\varphi_{\mathrm{m}}$. Поэтому для каждых конкретных значений констант $c$ и $a$ значение относительной влажности воздуха $\varphi_{\mathrm{m}}$ следует определять численным методом.

\section{Сопоставление результатов обработки изотермы сорбции по различным методам}

С целью сопоставления двух вышеизложенных методов по этим методам были обработаны изотермы сорбции следующих восьми широко применяемых строительных материалов: цементного камня, тяжёлого бетона, керамзитобетона, азеритобетона, красного кирпича, цементно-песчаного раствора и пенобетона. Эти изотермы были получены на вакуумной сорбционной установке при температуре $+20^{\circ} \mathrm{C}$. Для сопоставления были обработаны изотермы, полученные вакуумным методом, так как этот метод по сравнению с эксикаторным методом позволяет:

- более точно определить значения равновесной сорбционной влажности;

- получить большее число точек на изотерме.
Эти преимущества вакуумного метода особенно важны при исследовании равновесной сорбционной влажности при малых значениях относительной влажности воздуха.

С. Грег и К. Синг [12] на основе анализа результатов исследования адсорбции паров азота на различных катализаторах, хлориде натрия, кремнезёме и окиси алюминия, а также адсорбции паров криптона, изобутана и фтордихлорметана на серебряной фольге сделали вывод, что уравнение БЭТ применимо для анализа изотермы сорбции только в диапазоне относительных давлений адсорбата от 0,05 до 0,35. Такой же вывод сделан С. Брунауэром в его классической монографии [7]. В.Г. Гагариным [8] на основе применения $\mathrm{N}$-метода для анализа изотермы сорбции азота [13], аргона [14], кислорода [15] и водяного пара [16] сделал вывод, что N-метод применим для анализа изотермы сорбции строительных материалов в диапазоне относительной влажности воздуха от 0,05 до 0,5. При значениях относительной влажности воздуха больших, чем 0,5, в строительных материалах может иметь место капиллярная конденсация паров воды, а уравнение (10), положенное в основу N-метода, выведено без учёта возможности капиллярной конденсации адсорбата в порах адсорбента. Таким образом, основное преимущество $\mathrm{N}$-метода по сравнению с методом, основанным на уравнении БЭТ, заключается в том, что N-метод позволяет получить более точную информацию об адсорбционных характеристиках исследуемого материала, так как эту информацию получают путём обработки большего числа экспериментально полученных значений равновесной сорбционной влажности материала.

В таблице представлены результаты обработки изотерм сорбции вышеперечисленных строительных материалов по БЭТ- и N-методам. По БЭТ-методу были обработаны участки изотерм сорбции, соответствующие диапазону изменения относительной влажности воздуха $\varphi=0,05 \ldots 0,35$, а по N-методу $\varphi=0,05 \ldots 0,5$. Следует отметить , что результаты определения константы с и ёмкости монослоя $w_{\mathrm{m}}$ по $\mathrm{N}$-методу фактически не зависят от того, обрабатывался ли участок изотермы, соответствующий диапазону $\varphi=0,05 \ldots 0,35$ или $\varphi=0,05 \ldots 0,5$. Так, для тяжёлого бетона $\left(\gamma_{\mathrm{o}}=2440 \mathrm{\kappa r} / \mathrm{m}^{3}\right)$ в первом случае $c$ $=18,8, w_{\mathrm{m}}=0,0150 \mathrm{\kappa} г / \mathrm{\kappa} г$, а во втором $c=17,9, w_{\mathrm{m}}=0,0159 \mathrm{\kappa г} /$ кг. Что свидетельствует о высокой надёжности результатов, получаемых при обработке изотерм сорбции по N-методу.

Из данных таблицы следует, что значения константы $c$, определённые по N-методу для всех исследованных материалов, меньше, чем значения этой константы, определённые по БЭТ-методу. Для красного кирпича, цементно-песчаного раствора и пенобетона значение константы с, определённое по N-методу, в 2-3 раза меньше её значения, определённого по БЭТ-методу. Для остальных исследованных материалов это различие невелико. Эти различия могут быть объяснены тем, что численное значение константы с, определённое по $\mathrm{N}$-методу [см. формулу (16)], зависит только от значения углового коэффициента $a_{\mathrm{N}}$ линейной регрессии, который 
представляет собой тангенс угла наклона графика линейной регрессии к оси абсцисс. Поэтому даже небольшие изменения угла графика линейной регрессии могли привести к значительным изменениям вычисляемого значения константы $c$.

С другой стороны, эти различия в численных значениях констант $c$, определённых по этим двум методам, могут быть также объяснены ещё и тем, что численное значение константы $c$, определённое по БЭТ-методу [см. формулу (8)], про-

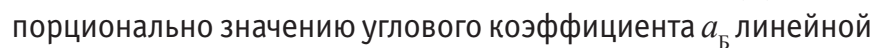
регрессии. Для исследованных материалов при обработке их изотерм по БЭТ-методу значения углового коэффициента менялись от 48,8 (асбестоцемент) до 361 (красный кирпич). Таким образом, угол наклона графика линейной регрессии в этом случае был близок к $\pi / 2$. Следовательно даже небольшие изменения этого угла могли привести к значительным изменениям вычисленного по БЭТ-методу значения константы $c$.

Из таблицы следует, что значения ёмкости монослоя $w_{\mathrm{m}}$ и, как следствие, значения удельной поверхности $A$, а также значения относительной влажности воздуха $\varphi_{\mathrm{m}}$, определённые по БЭТ- и N-методам, различаются не более, чем на 10\%, то есть незначительно. Для шести исследованных материалов значения ёмкости монослоя $w_{\mathrm{m}}$, определённые по N-методу, больше, чем значения этого показателя, определённые по БЭТ-методу, а по двум материалам - меньше. Таким образом, можно утверждать, что различия между значениями ёмкости монослоя $w_{\mathrm{m}}$, определёнными по этим двум методикам, носят случайный характер.

Подставив вычисленные значения константы $c$ и ёмкости монослоя $w_{\mathrm{m}^{\prime}}$ в формулы (1) и (17), можно получить уравнения начальных участков изотерм сорбции исследованных строительных материалов при температуре $+20^{\circ} \mathrm{C}$, определенные по БЭТ-методу $(\varphi=0,05-0,35)$ и N-методу $(\varphi=0,05-0,50)$. В качестве примера приведём эти уравнения для двух исследованных материалов: красного кирпича и керамзитобетона.

Таблица. Результаты обработки изотерм сорбции по БЭТ-методу и $\mathrm{N}$-методу

\begin{tabular}{|c|c|c|c|c|c|}
\hline Материал & Метод & $\begin{array}{c}\text { Констан- } \\
\text { та с }\end{array}$ & $\begin{array}{c}\text { Емкость } \\
\text { монослоя } \\
w_{m}, \text { кr/kr }\end{array}$ & $\begin{array}{l}\text { Относитель- } \\
\text { ная влаж- } \\
\text { ность возду- } \\
\text { ха } \varphi_{\mathrm{m}}, \text { Па/Па }\end{array}$ & $\begin{array}{c}\text { Удельная по- } \\
\text { верхность } A, \\
10^{3} \mathrm{~m}^{2} / \mathrm{kr}\end{array}$ \\
\hline \multirow{2}{*}{ Цементный камень } & БЭТ & 111 & 0,0118 & 0,087 & 45 \\
\hline & $\mathrm{N}$ & 74,4 & 0,0115 & 0,094 & 44 \\
\hline \multirow{2}{*}{$\begin{array}{l}\text { Керамзитобетон } \\
\gamma_{0}=1000 \mathrm{kr} / \mathrm{M}^{3}\end{array}$} & БЭТ & 94,3 & 0,0105 & 0,093 & 40 \\
\hline & $\mathrm{N}$ & 87,6 & 0,0116 & 0,089 & 45 \\
\hline \multirow{2}{*}{$\begin{array}{l}\text { Тяжелый бетон } \\
\gamma_{0}=2440 \mathrm{kr} / \mathrm{M}^{3}\end{array}$} & БЭТ & 26,6 & 0,0153 & 0,17 & 58 \\
\hline & $\mathrm{N}$ & 17,9 & 0,0159 & 0,17 & 61 \\
\hline \multirow{2}{*}{$\begin{array}{l}\text { Азеритобетон } \\
\gamma_{0}=800 \mathrm{kr} / \mathrm{m}^{3}\end{array}$} & БЭТ & 16,8 & 0,00611 & 0,20 & 23 \\
\hline & $\mathrm{N}$ & 13,7 & 0,00608 & 0,20 & 23 \\
\hline \multirow{2}{*}{$\begin{array}{l}\text { Асбестоцемент } \\
\text { автоклавный }\end{array}$} & БЭТ & 24,1 & 0,0197 & 0,17 & 75 \\
\hline & $\mathrm{N}$ & 22,5 & 0,0208 & 0,16 & 79 \\
\hline \multirow{2}{*}{$\begin{array}{l}\text { Красный кирпич } \\
\gamma_{0}=1890 \mathrm{kr} / \mathrm{M}^{3}\end{array}$} & БЭТ & 51,9 & 0,00272 & 0,12 & 10 \\
\hline & $\mathrm{N}$ & 24,0 & 0,00282 & 0,15 & 11 \\
\hline \multirow{2}{*}{$\begin{array}{l}\text { Цементно-песчаный } \\
\text { раствор 1:3, В/ЦL=0,55 }\end{array}$} & БЭТ & 34,0 & 0,00708 & 0,15 & 27 \\
\hline & $\mathrm{N}$ & 11.6 & 0,00805 & 0,21 & 31 \\
\hline \multirow{2}{*}{$\begin{array}{l}\text { Пенобетон } \\
\gamma_{0}=680 \mathrm{kr} / \mathrm{M}^{3}\end{array}$} & БЭТ & 59,6 & 0,0141 & 0,12 & 54 \\
\hline & $\mathrm{N}$ & 20,6 & 0,0156 & 0,16 & 59 \\
\hline
\end{tabular}

$$
\begin{aligned}
& \text { Красный кирпич } \\
& \text { БЭТ-метод }-\quad \gamma_{p}(\varphi)=\frac{0,00272 \varphi}{(1-\varphi)(0,0193+0,981 \varphi)}, \\
& \text { N-метод - } \quad w_{p}(\varphi)=\frac{0,0688 \varphi}{1+23,4 \varphi}\left(\frac{3,22}{-\ln \varphi}\right)^{0,392} .
\end{aligned}
$$

Керамзитобетон

$$
\left(\gamma_{\mathrm{o}}=1000 \mathrm{\kappa r} / \mathrm{m}^{3}\right) \text { : }
$$

$$
\begin{array}{ll}
\text { БЭТ-метод - } & w_{p}(\varphi)=\frac{0,0105 \varphi}{(1-\varphi)(0,0106+0,089 \varphi)}, \\
\mathrm{N} \text {-метод - } & w_{p}(\varphi)=\frac{1,02 \varphi}{1+86,6 \varphi}\left(\frac{3,22}{-\ln \varphi}\right)^{0,392} .
\end{array}
$$

Основное преимущество $\mathrm{N}$-метода по сравнению с БЭТметодом заключается в том, что N-метод позволяет получить более точную информацию об адсорбционных характеристиках исследуемого материала, так как при его применении эту информацию получают путём обработки большего числа экспериментально полученных значений равновесной сорбционной влажности этого материала. Особенно это преимущество важно при исследовании адсорбционных характеристик строительных материалов на основании изотерм сорбции, полученных эксикаторным методом, так как в этом случае число экспериментальных точек на изотерме, как правило, не превышает шести.

\section{Лuтература}

1.Умнякова, Н.П. Развитие методов нормирования теплозащиты энергоэффективных зданий / Н.П. Умнякова, И.Н. Бутовский, А.Г. Чеботарёв // Жилищное строительство. - 2014. - № 7. - С. 19-21.

2. Шеина, С.Г. Методика выбора энергоресурсосберегающих решений на этапе эксплуатации жилых зданий / С.Г. Шеина, Е.Н. Миненко // Бюллетень строительной техники. - 2017. - № 6 (994). - С. 54-55.

3. Пастушков, П.П. Использование расчётного определения эксплуатационной влажности теплоизоляционных материалов / П.П. Пастушков, Н.В. Павленко, Е.В. Коркина // Строительство и реконструкция. - 2015. - № 4 (60). - С. 168-172.

4. Гагарин, В.Г. Определение расчетной влажности строительных материалов / В.Г. Гагарин, П.П. Пастушков // Промышленное и гражданское строительство. - 2015. - № 8. - С. 28-33

5. Гагарин, В.Г. К вопросу о назначении расчетной влажности строительных материалов по изотерме сорбции / В.Г. Гагарин, П.П. Пастушков, Н.А Реутова // Строительство и реконструкция. - 2015. - № 4 (60). - С. 152-155.

6. Совершенствование теплотехнического проектирования зданий в климатических условиях Республики Саха (Якутия) / Н.П. Умнякова, И.Н. Бутовский, А.Г. Чеботарёв, 0.И. Матвеева // Жилищное строительство. - 2015. - № 7. - С. 12-17.

7. Брунауэр, С. Адсорбция паров и газов. Т. 1. Физическая адсорбция / С. Брунауэр ; пер. с англ. ; Под ред. акад. М. М. Дубинина. - М. : Гос. изд-во иностр. лит., 1948. - 482 с.

8. Гагарин, В.Г. О модификации t-метода для определения удельной поверхности макро- и мезопористых адсорбентов / В.Г. Гагарин // Журн. физ. химии. - 1985. - Т. 59. - № 5. -С. 1838-1839. 
9. Литвинова, Т.А. Адсорбция паров воды капиллярнопористыми телами / Т.А. Литвинова // Производственный НИИ по инженерным изысканиям в строительстве. - М., 1984. - Вып. 44. - C. 17-21

10. Whalen, J.W. Adsorption of water vapour on quartz powder /J.W. Whalen // J. Phys. Chem. -1993. - Vol. 98. - P. 1676-1680.

11. Скоблинская, Н.Н. Модифицированный метод измерения изотерм сорбции-десорбции воды / Н.Н. Скоблинская // М. : ПЭМ ВНИИС. - М., 1983.

12. Грег, С. Адсорбция, удельная поверхность, пористость / С. Грег, Л. Синг. - М., 1984. - 527 с.

13. Lippens, B.C. Studies on pore in catalysts. 5 . The t-method / B.C. Lippens, J.H. Boer De // J. Catalisis. - 1965. - Vol. 4. - № 3. - P. 319-323.

14. Payne, D.A. Comparison of argon and nitrogen adsorption isotherms on porous and nonporous hydroxylated silica / D.A. Payne, K.S.W. Sing, D.H. Turk // J. Coll. Int. Sci. - 1973. - Vol. 43. - № 2. - P. 287-293.

15. Pore structure analysis by oxygen adsorption. I. t-curves and methods of analysis / Hanna K.M., Older I., Brunauer S. [et al.] // J. Coll. Int. Sci. - 1973. - Vol. 45 - № 1. - P. 27-37.

16. Sheck, T. Measurements of pore size distribution of porous materials / Sheck T., Oinonen H. - Helsinki, 2014. - 41 p.

\section{References}

1. Umnyakova N.P. Razvitie metodov normirovaniya teplozashchity energoeffektivnykh zdanii [Development of methods for rationing thermal protection of energy-efficient buildings]. Zhilishchnoe stroitel'stvo [Housing construction], 2014, no. 7, pp. 19-21. (In Russ.)

2. Sheina S.G., Minenko E.N. Metodika vybora energoresursosberegayushchikh reshenii na etape ekspluatatsii zhilykh zdanii [The methodology of choosing energy-saving solutions at the stage of operation of residential buildings]. Byulleten stroitel'noi tekhniki [Bulletin of construction equipment], 2017, no. 6 (994), pp. 54-55.

3. Pastushkov P.P., Pavlenko N.V., Korkina E.V. Ispol'zovanie raschetnogo opredeleniya ekspluatatsionnoi vlazhnosti teploizolyatsionnykh materialov [Using the calculated determination of the operational humidity of heat-insulating materials]. Stroitel'stvo i rekonstruktsiya [Construction and reconstruction], 2015, no. 4 (60), pp. 168-172.

4. Gagarin V.G., Pastushkov P.P. Opredelenie raschetnoi vlazhnosti stroitel'nykh materialov [Determination of the estimated humidity of building materials]. Promyshlennoe $i$ grazhdanskoe stroitel'stvo [Industrial and civil engineering], 2015, no. 8, pp. 28-33.

5. Gagarin V.G., Pastushkov P.P., Reutova N.A. K voprosu o naznachenii raschetnoi vlazhnosti stroitel'nykh materialov po izoterme sorbtsii [To the question of the designation of the estimated moisture content of building materials by the sorption isotherm]. Stroitel'stvo i rekonstruktsiya [Construction and reconstruction], 2015, no. 4 (60), pp.152-155.

6. Umnyakova N.P., Butovskii I.N., Chebotarev A.G., Matveeva 0.I. Sovershenstvovanie teplotekhnicheskogo proektirovaniya zdanii v klimati-cheskikh usloviyakh Respubliki Sakha (Yakutiya) [Improving the thermal engineering design of buildings in the climatic conditions of the Republic of Sakha (Yakutia)]. Zhilishchnoe stroitel'stvo [Housing construction], 2015, no. 7, pp. 12-17.

7. Brunauer S. Adsorbtsiya parov i gazov. T. 1. Fizicheskaya ad-sorbtsiya [Adsorption of vapors and gases. Vol. 1. Physical adsorption], Trans. from English, Acad. M.M. Dubinin (ed.). Moscow, State. Publishing House of Foreignlit. Publ., 1948, 482 p.

8. Gagarin V.G. 0 modifikatsii t-metoda dlya opredeleniya udel'noi poverkhnosti makro- i mezoporistykh adsorbentov [0n the modification of the t-method for determining the specific surface area of macro- and mesoporous adsorbents]. Zhurn. fiz. khimii [Journal of Physical Chemistry], 1985, vol. 59, no. 5, pp. 1838-1839.

9. Litvinova T.A. Adsorbtsiya parov vody kapillyarno-poristymi telami. V kn. Proizvodstvennyi NII po inzhenernym izyskaniyam v stroitel'stve [Industrial Research Institute for Engineering Surveys in Construction]. Moscow, 1984, Iss. 44, pp. 17-21.

10. Whalen J.W. Adsorption of water vapour on quartz powder. J. Phys. Chem.,1993, Vol. 98, pp. 1676-1680.

11. Skoblinskaya N.N. Modifitsirovannyi metod izmereniya izo-term sorbtsii-desorbtsii vody [A modified method for measuring the iso-therm of sorption-desorption of water]. Moscow, PEM VNIIS Publ., 2008.

12. Greg S., Sing L. Adsorbtsiya, udel'naya poverkhnost', poristost' [Adsorption, specific surface area, porosity]. Moscow, 1984, 527 p.

13. Lippens B.C., De Boer J.H. Studies on pore in catalysts. 5. The t-method. J. Catalisis, 1965, Vol. 4, no. 3, pp. 319-323.

14. Payne D.A., Sing K.S.W., Turk D.H. Comparison of argon and ni-trogen adsorption isotherms on porous and nonporous hydroxylated silica. J. Coll. Int. Sci., 1973, Vol. 43, no. 2, pp. 287-293.

15. Hanna K.M., Older I., Brunauer S., Hagymassy J., Bodor E. Pore structure analysis by oxygen adsorption. I. t-curves and methods of analysis. J. Coll. Int. Sci., 1973, Vol. 45, no. 1, pp. 27-37.

16. Sheck T., Oinonen H. Measurements of pore size distribution of porous materials. Helsinki, 2014, 41 p.

Киселёв Игорь Яковлевич (Москва). Доктор технических наук, старший научный сотрудник, почётный член РААСН. Учёный секретарь ФГБУ «Научно-исследовательский институт строительной физики Российской академии архитектуры и строительных наук» (127238, Локомотивный, проезд, 21. НИИСФ РААСН). Эл. почта: ikiselyov@bk.ru.

Kiselev Igor Y. (Moscow). Doctor of Technical Sciences, Senior Researcher, Honorary Member of RAACS. Scientific Secretary at the Scientific-Research Institute of Building Physics of RAACS (21 Lokomotivny proezd, Moscow, 127238. NIISF RAASN). E-mail: ikiselyov@bk.ru. 